\title{
The Use of FIB for the Preparation of a Structure Analogous to the Natural Sur- face and Its Replication by Selected Types of Polymers
}

\author{
Dora Kroisová1 ${ }^{1}$ Tomáš Šamořil ${ }^{2}$, Štěpánka Dvořáčková ${ }^{1}$ \\ ${ }^{1}$ Faculty of Mechanical Engineering, Technical University of Liberec. Studentska 2, 46117 Liberec. Czech Repub- \\ lic.E-mail: dora.kroisova@tul.cz, stepanka.dvorackova@tul.cz \\ 2Tescan Orsay Holding, Libusina 21, 62300 Brno. Czech Republic. E-mail: tomas.samoril@tescan.com
}

The experiments aimed to verify the use of a focused ion beam (FIB) to create structures analogous to the natural surface and then use the two-step imprinting method to prepare replicas of the surface created by FIB. The surface of the petal leaves of pansy (Viola $x$ wittrockiana) was selected as a natural surface pattern. The TESCAN AMBER FIB-SEM microscope was used for the preparation of an analogous surface. The molds for the replication process were made of President Light Body elastomer. PVB, PVA, CMC, PCL, EP were used for the preparation of polymer replicas. Scanning electron microscope was used to evaluate the quality of the prepared polymer replicas. The dimensions of the replicated convex and concave structures were $25 \times 20 \mu \mathrm{m}$. The polymers showed a different ability to copy the structure in detail. For PVA, CMC, and PCL, surface structuring at the nanometer level was described in this study. Due to the dimensions of the cones of convex and concave structures, the color was observed on all samples.

Keywords: Natural Surface Structures, Focused Ion Beam, Replication, Scanning Electron Microscopy

\section{Introduction}

In recent years, natural surface structures have attracted attention from several points of view - super-hydrophobicity, abrasion resistance, temperature control options, antibacterial behavior, reflection, or absorption of sunlight. The most common shape found on the surface of natural objects is a cone. Cones have different dimensions and on the surfaces of the cones further surface structures can be found. The dimensions of the base of the cones as well as their height range from hundreds of nanometers to tens of micrometers. Conical structures that look more like fibers are called trichomes. The dimensions, structuring, shape, and layout of the cones are related to the function they are performing on the surface of the object. For example, in plants, the nature of the surface cones is related to the appearance, wettability, and reflectivity of the surface. For the potential use of such structures in practice, it is necessary to verify the functionality of their synthetic analogies. [1,2]

The simplest and most common way to artificially prepare such structures is the two-step imprint method. The natural surface serves as a pattern on which a layer of elastomer is applied. After removing the crosslinked elastomer from the natural surface, a mold is formed which is used to make a subsequent replica. The disadvantage of this method is that it is always necessary to work with fresh material, because of water evaporation. Especially in the case of plant samples, there is a degradation of the structures due to the loss of their true shape. There are other possibilities for imitating natural surfaces, however, they are time-consuming, financially, and technologically demanding. These include methods such as chemical vapor deposition, etching, lithography, layer by layer, spin coating, sol-gel dip coating, 3D nano printing. One of the most promising ways to create an analogy of the natural surface is scanning electron microscope equipped with focused ion beam (FIB-SEM). FIBSEM is usually used for imaging of the structures of the different materials on a micrometer and nanometer scale for micro-, nano-preparation of the structures. $[2,3,4,5,6,7,8]$

The content of the presented text is experimental verification of the possibility of using a focused ion beam for the preparation of surface structures analogous to natural patterns and the use of the two-step imprinting method to obtain replicas of the surface. Different polymeric materials (water-alcohol-soluble polymers, amorphous and crystallizing polymers) were used for the experiment to describe their ability to replicate the surface formed by FIB.

\section{Materials and Methods}

A silicon wafer was used to prepare the synthetic surface by FIB milling. A two-component elastomeric polymer, President Light Body (Coltene Wahledent, Hamburg), was used for the replication process. Polymers were used for the preparation of replicas: polyvinyl alcohol (Merck KGaA) and polyvinyl butyral 
(Merck KGaA), carboxymethylcellulose (Likochem.cz), polycaprolactone (Merck KGaA), epoxy resin EnviPOXY ${ }^{\circledR}$ (Spolchemie, a. s.). The selection includes water and alcohol soluble polymers, biodegradable polymers, amorphous and semi-crystalline polymers. For the experiments, the surface of the garden pansy flower (Viola $x$ wittrockiana) was selected as a pattern of the natural surface with conical structures. The cones on the surface of the petal leaves have dimensions at the base of $30-40 \mu \mathrm{m}$ and a height of 40 - $50 \mu \mathrm{m}$. The base of the cones has a hexagonal shape, the structural folds on the surface of the cones are usually in the range of $300-400 \mathrm{~nm}$, the same is their thickness. This natural surface exhibits both superhydrophobic behavior and minimal surface reflectivity. Based on the characteristics of this structure, model design for the focused ion beam milling process was developed.

TESCAN AMBER FIB-SEM equipped with Ga ion source was used for FIB milling process. The energy of ions was $30 \mathrm{keV}$. The structures were designed and draw in the lithographic module DrawBeam - Fig. 1. Structures were created with ion current $50 \mathrm{pA}$ into silicon sample. The total surface preparation time of the convex and concave structures, each measuring 25 $\mu \mathrm{m} \times 20 \mu \mathrm{m}$, was 12 hours.

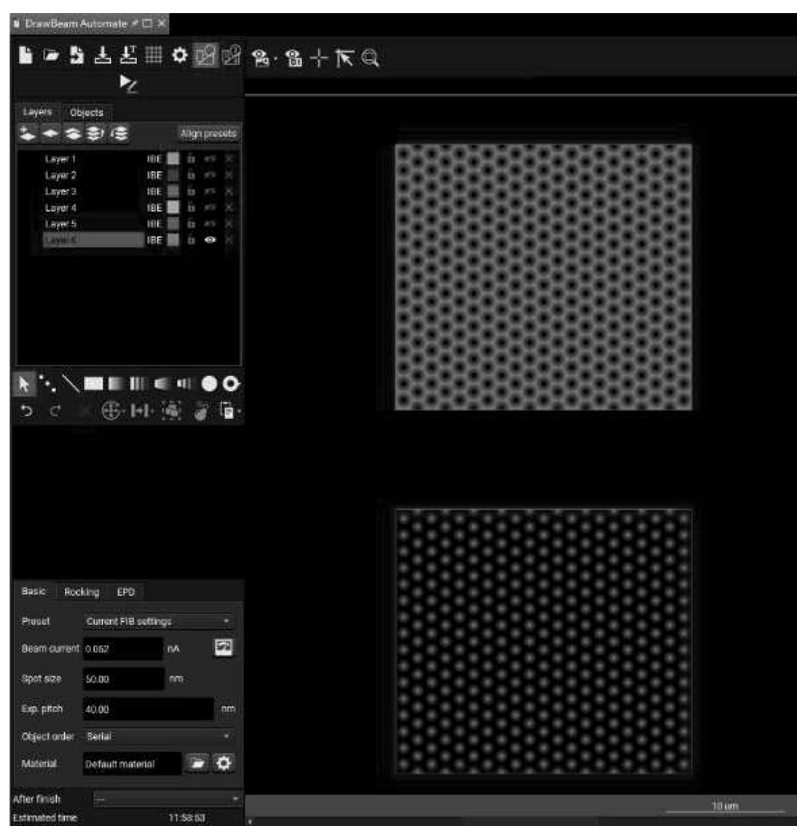

Fig. 1 DrawBeam patterns used for the fabrication convex and concave structures by local FIB milling. TESCAN AMBER FIB-SEM

The two-step imprinting method consisted of applying a few millimeters thick layer of elastomer to a silicon sample and then loading this system (up to $5 \mathrm{~N}$ ) for better flow of the elastomer into microscopic and submicroscopic structures. After crosslinking the elastomer for several minutes, the elastomeric layer was removed from the sample. This created the so-called first in other words negative replica. This served as a mold for the preparation of second in other words positive replicas of the pattern created by FIB, and replicas were prepared from these types of polymers. The replication process took place at atmospheric pressure, normal laboratory temperature, and without surface loading. Only in the case of polycaprolactone was an elevated temperature used to melt the polymer and load the sample (up to $5 \mathrm{~N}$ ) during polymer crystallization.

Characterization of plant pattern structures prepared silicon models, silicone molds, and polymer replicas were performed on scanning electron microscopes ZEISS ULTRA PLUS and TESCAN MIRA3 and TESCAN AMBER FIB - SEM. All evaluated samples were attached to the aluminium target with double-sided carbon adhesive tape. Both natural and polymer samples were sputtered with a layer of Pt-Pd (Quorum Q150R ES) with a thickness of 2 - $4 \mathrm{~nm}$. Samples of the structure prepared on a silicon wafer by a focused ion beam were not surface treated.

\section{Results}

For the preparation of synthetic surfaces by the process of milling with a focused beam of $\mathrm{Ga}$ ions, the surface used in the epidermal layer of the petals of pansy flowers (Viola $\times$ wittrockiana) was used as a model. This surface is formed by structured cones - Fig. 2. Based on this surface, a model was created - Fig. 1, which was used for the two-step imprinting process. Two structures were created by this process - Fig. 3, Fig. 4, Fig. 5. The difference between the natural model and the structure prepared by FIB was in the dimensions of the cones and the character of their surface. On the surface of the natural sample, the cones had dimensions at the base of $30-40 \mu \mathrm{m}$ and a height of 40 - $50 \mu \mathrm{m}$ and were longitudinally structured - Fig. 2 . Due to the considerable time required to produce such a surface on a silicon wafer, which would at the same time have sufficient dimensions for further experiments, a surface analogous in shape but with smaller cone dimensions and without surface structuring was selected. The base of the cones of the proposed model had a diameter of $1 \mu \mathrm{m}$, the height of the cones was also $1 \mu \mathrm{m}$. The dimensions at the tops of the cones were around $200 \mathrm{~nm}$. The size of both samples was designed to be about $20 \times 20 \mu \mathrm{m}$. This model was used to produce the structure in a silicon wafer.

Scanning electron microscope images show that the size of the prepared samples is at the level of tens of micrometers - Fig. 3. Two types of patterns were created for the experiment, in one pattern the conical structure protrudes from the surface and is hereinafter referred to as convex, in the other pattern, on the contrary, the structure is referred to as concave. The quality of the created structures is high - Fig. 4 and Fig. 5. 
Fig. 5 also shows the surface of the cones on the silicon wafer. The incident radiation was reflected on the silicon wafer, the silicone mold, and on all prepared polymer replicas, and the structures showed color due to the dimensions of the cones, respectively the nature of the formed structure. This made it easier to identify, under an optical microscope, the location where a replica of a structure that appeared to be a colored rectangular shape was formed in the area of the entire sample.

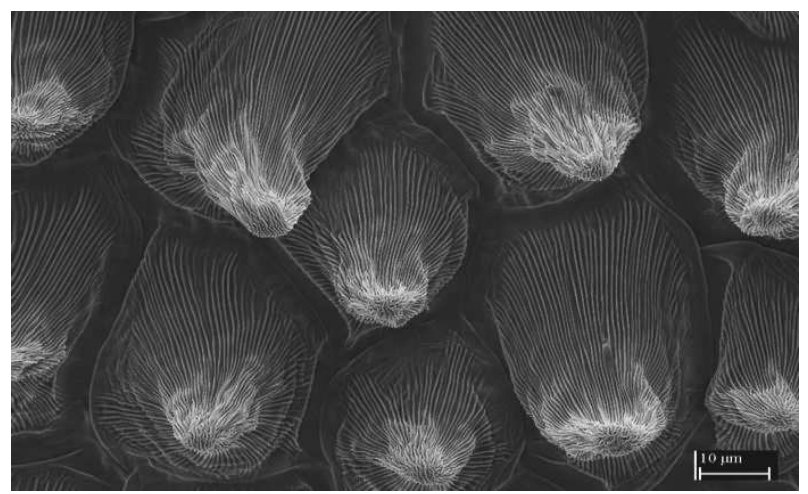

Fig. 2 The natural pattern used to design the structure made by FIB The cones in the epidermal layer of the petal leaves of the garden pansy are deformed due to the drying of the plant. ZEISS ULTRA PLUS

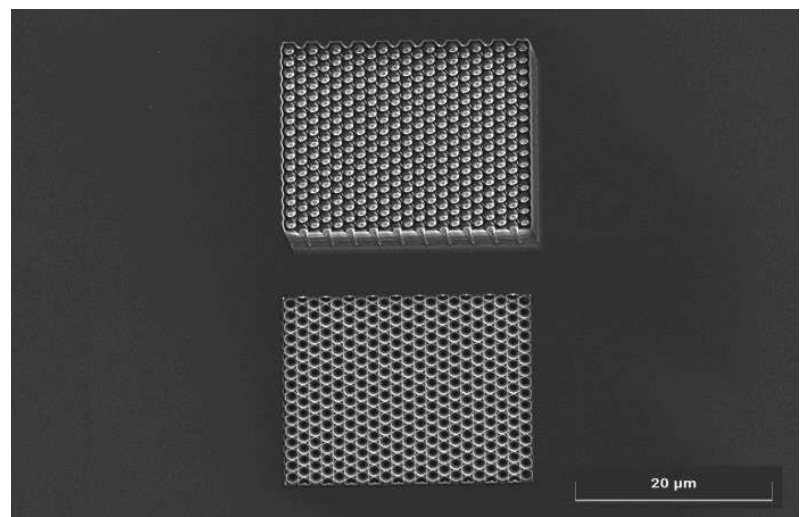

Fig. 3 The convex and concave structure was created by FIB on a silicon wafer - the analogy of a natural surface. TESCAN AMBER FIB-SEM

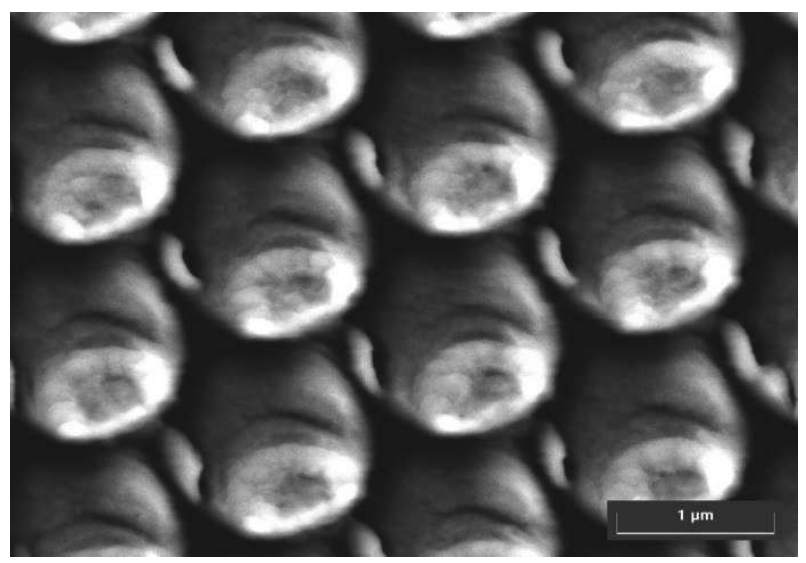

Fig. 4The convex structure was created by FIB on a silicon wafer - analogy of a natural surface detailed image. TESCAN AMBER FIB-SEM

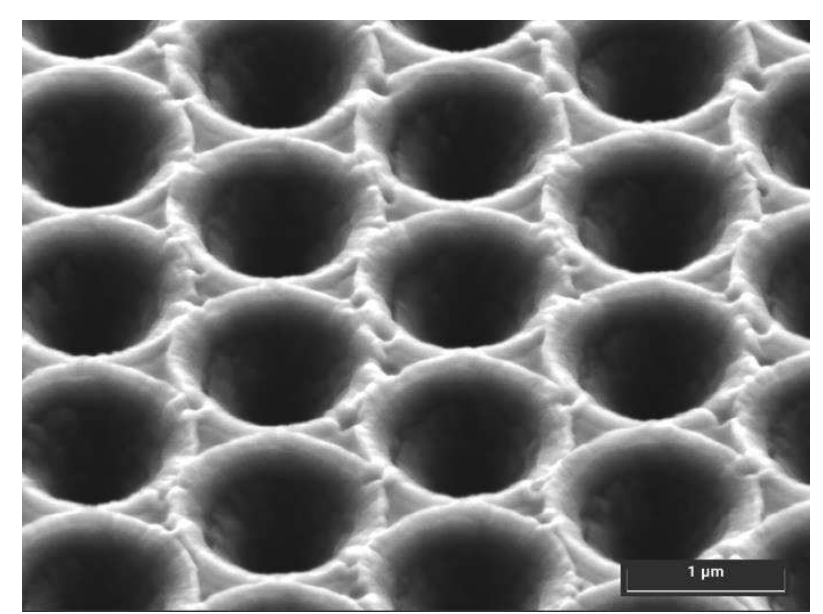

Fig. 5 The concave structure was created by FIB on a silicon wafer - analogy of a natural surface detailed image. TESCAN AMBER FIB-SEM

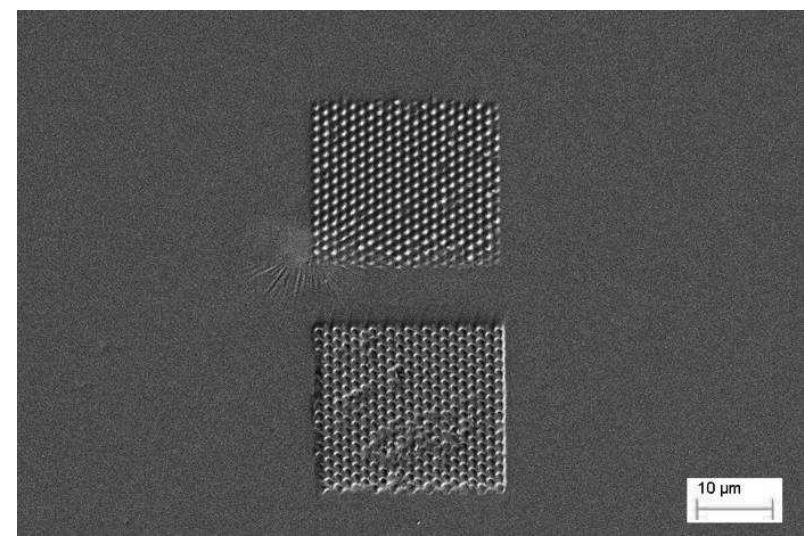

Fig. 6 The elastomeric mold formed as an imprint of the surface of a silicon wafer. ZEISS ULTRA PLUS

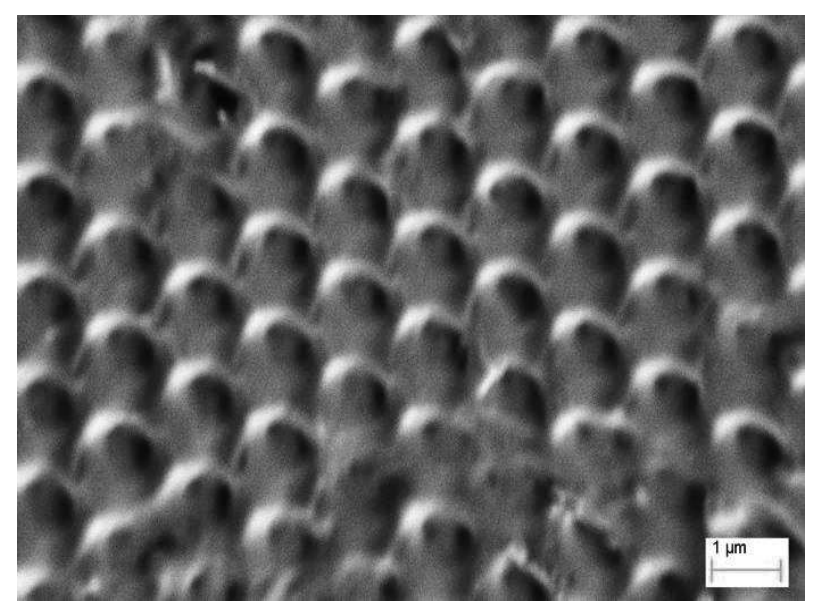

Fig. 7 The elastomeric mold formed as an imprint of the surface of a silicon wafer - detailed image ZEISS ULTRA PLUS

The elastomeric (silicone) mold formed by replication of the surface which was formed on the silicon wafer has both areas that perfectly copy the surface of the pattern and areas with defects - Fig. 6. A detailed representation of both types of patterns is shown in Fig. 7 and Fig. 8. Molds in the detailed view are not 
represented in high quality, because, despite the application of the Pt-Pd nanolayer, silicone samples were charged in the chambers of all used electron microscopes. However, the advantage of the silicone mold was its easy removal both from the silicon wafer and from samples of all selected polymers. For the replication process, 5 types of polymeric materials were used, which differed in their chemical composition, crystallization ability, and method of application to the structured surface of the silicone mold.

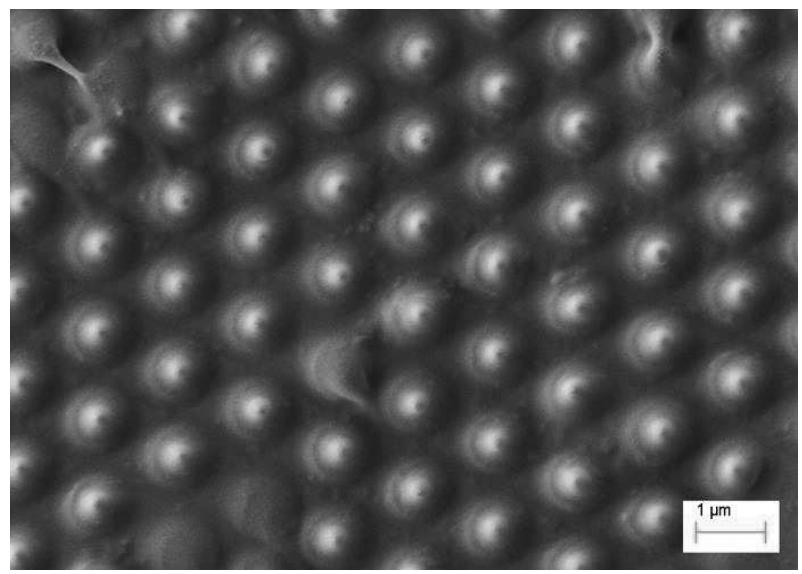

Fig. $\boldsymbol{8}$ The elastomeric mold formed as an imprint of the surface of a silicon wafer - detailed image ZEISS ULTRA PLUS

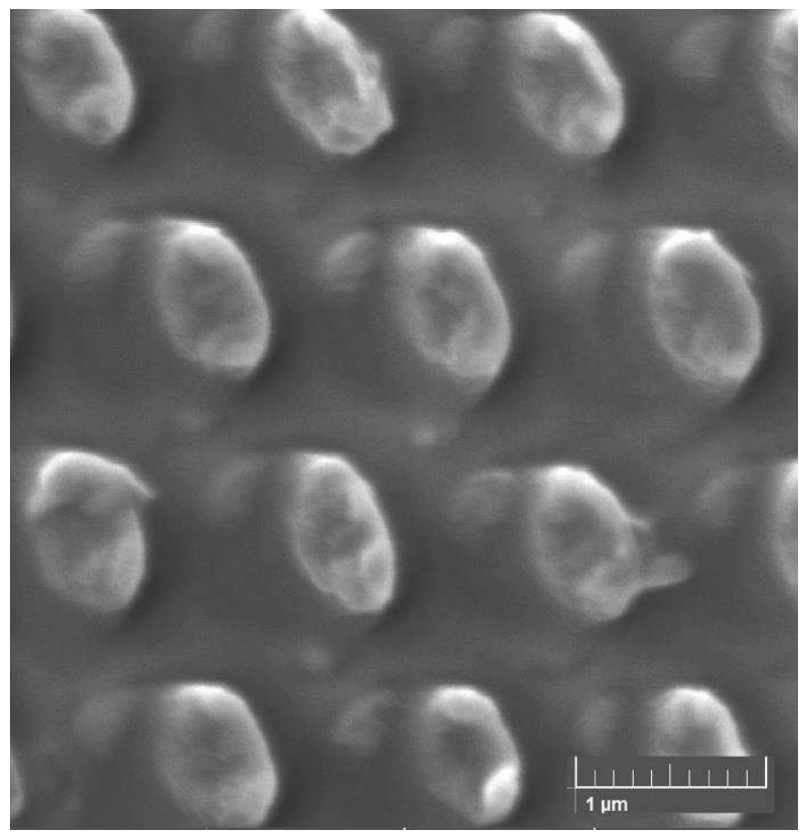

Fig. 9 The polyvinyl butyral (PVB) replica of pattern - convex conical structure - detailed image. TESCAN MIRA3

Polyvinyl butyral (PVB) is an alcohol-soluble polymer. It was applied to the silicone mold as a $10 \mathrm{wt} . \%$ alcohol solution. The PVB sample was left in the mold for 24 hours at a normal laboratory temperature of $22^{\circ}$ $\pm 2^{\circ} \mathrm{C}$ and normal atmospheric pressure. The sample was then removed from the mold. The thickness of the PVB samples was in the tenths of a millimeter.
From the scanning electron microscope images - Fig. 9 and Fig. 10 it can be seen this type of polymer can form a replica of the original pattern, in cases where the conical structure is convex and concave. In contrast to the pattern on the silicon wafer, the convex cones - Fig. 9 are smaller and the free spaces between the mini ones are larger. This can be explained by the fact that the alcohol evaporates from the sample and the cones are formed by the remaining polymer. Concave cones - Fig. 10 are copied well. However, their depth does not correspond to the depth of the pattern. The surface structure of the polymer cones is not visible in the images.

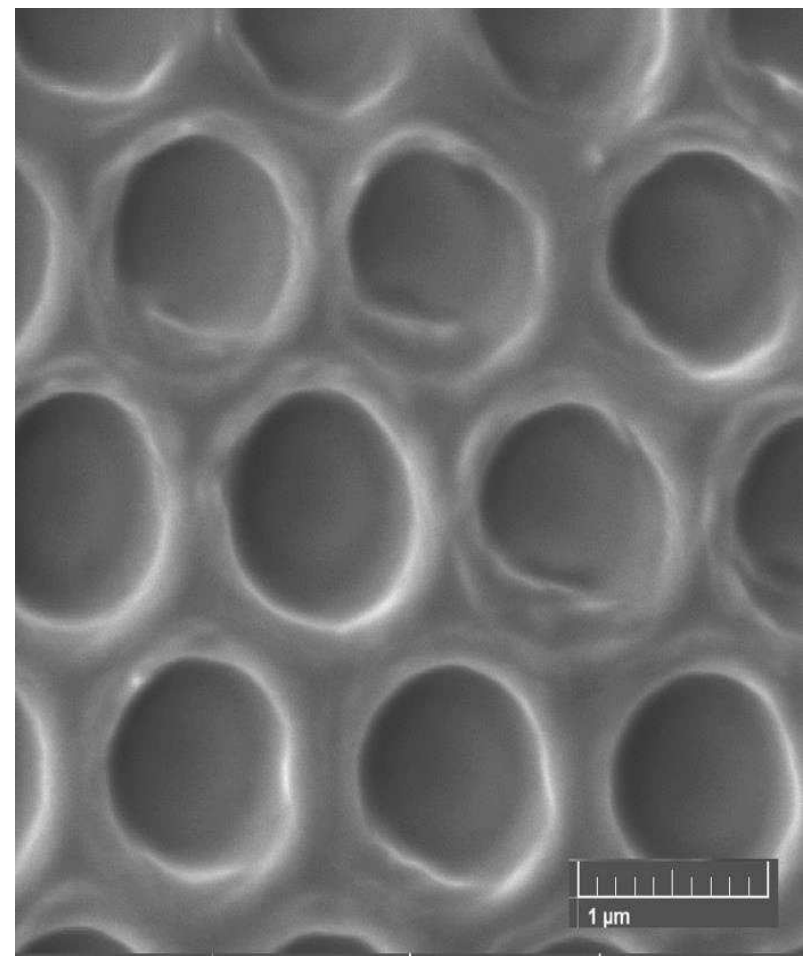

Fig. 10 The polyvinyl butyral (PVB) replica of pattern concave conical structure - detailed image. TESCAN $\operatorname{MIRA3}$

Polyvinyl alcohol (PVA) a water-soluble polymer was applied to the silicone mold as a $10 \mathrm{wt} . \%$ aqueous solution. The PVA sample was left in the mold for 24 hours at a normal laboratory temperature of $22^{\circ} \pm 2^{\circ} \mathrm{C}$ and normal atmospheric pressure. The sample was then removed from the mold. The thickness of the PVA samples was also in the tenths of a millimeter. From the scanning electron microscope images - Figs. 11, Fig. 12 it is evident that this type of polymer can create very high quality convex and concave structures, cones and their sizes correspond very well to the pattern on the silicon pattern. A new surface structure was found at convex cones - Fig. 11. This fact can be explained by the evaporation of water from the sample during the replication process, which takes place at atmospheric pressure and room temperature. Concave cones - Fig. 12 correspond to their pattern. 


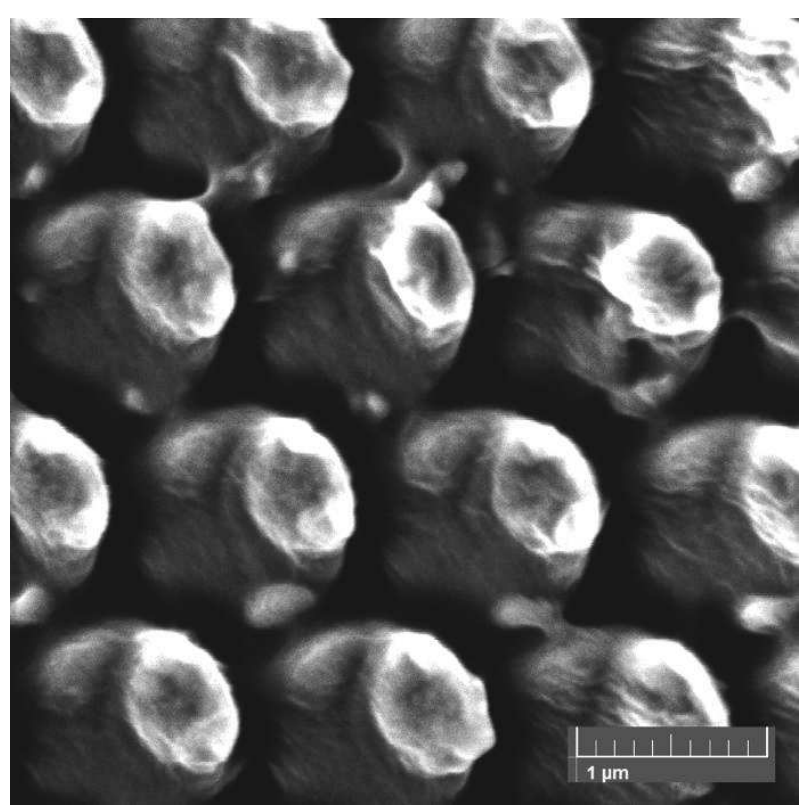

Fig. 11 The polyvinyl alcohol (PVA) replica of pattern - convex conical structure - detailed image. TESCAN MIRA3

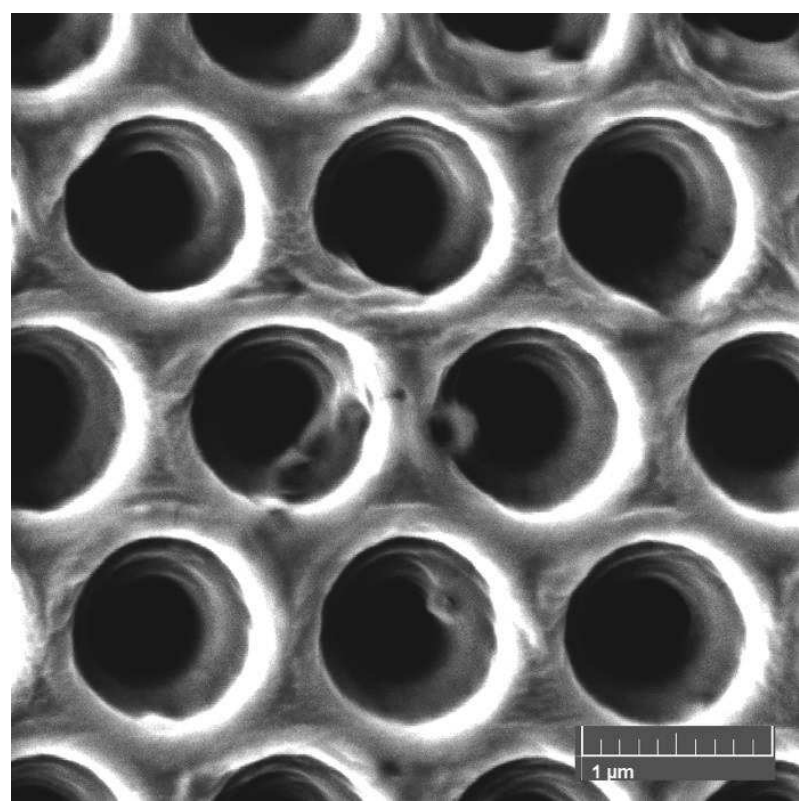

Fig. 12 The polyvinyl alcohol (PVA) replica of pattern concave conical structure - detailed image. TESCAN MIRA3

Carboxymethylcellulose (CMC) is a water-soluble polymer. It was applied to the silicone mold as a 10 wt.\% aqueous solution. The CMC sample was left in the mold for 24 hours at a normal laboratory temperature of $22^{\circ} \pm 2^{\circ} \mathrm{C}$ and normal atmospheric pressure. The sample was then removed from the mold. The thickness of the CMC samples was similar to the previous samples in tenths of a millimeter. From the scanning electron microscope images - Fig. 13, Fig. 14 it is evident that this type of polymer is also able to create high quality convex and concave replicas of structures, which by the arrangement of cones and their sizes correspond well to the original pattern on the silicon wafer. The surface of convex cones is surface-structured, in image - Fig. 13 depressions at the level of hundreds of nanometers are visible in detail. As in the previous cases, this fact can be explained by the evaporation of water from the sample in the replication process, which takes place at atmospheric pressure and room temperature. Concave cones - Fig. 14 do not reach the quality of the original pattern, there are visible surface structures, the explanation is analogous to convex cones.

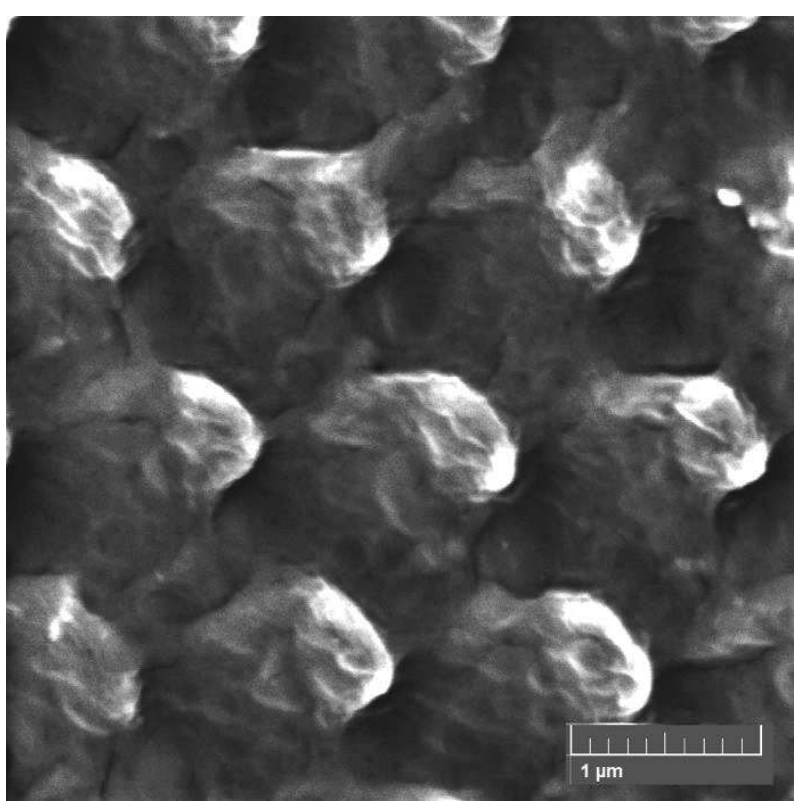

Fig. 13 The carboxymethylcellulose (CMC) replica of pattern - convex conical structure - detailed image. TESCAN MIRA3

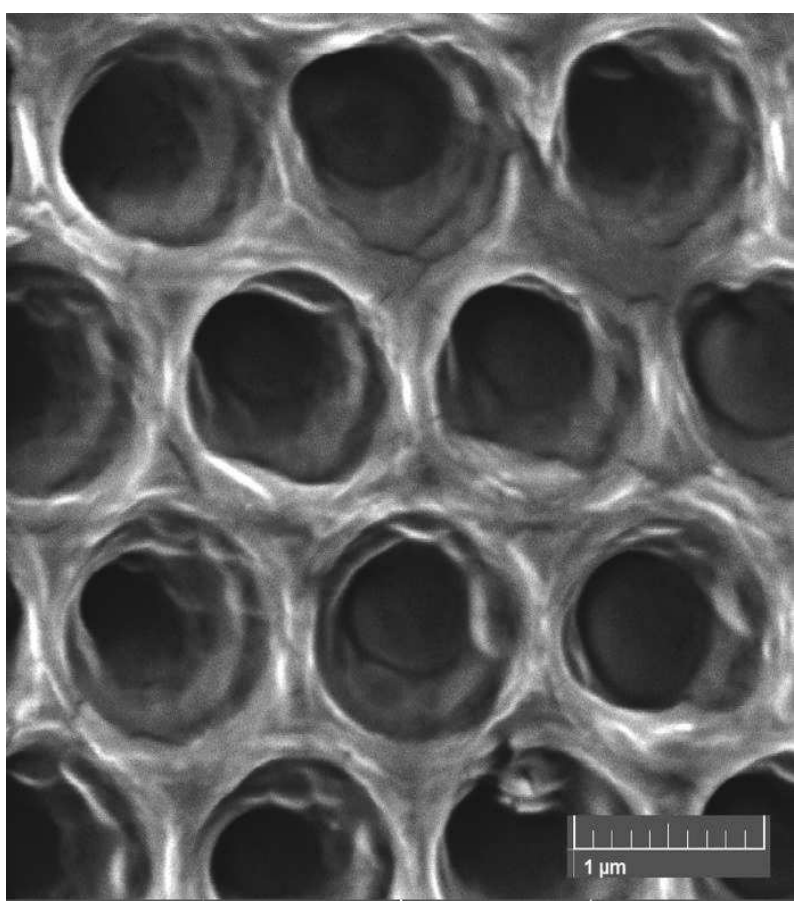

Fig. 14 The carboxymethylcellulose (CMC) replica of pattern - concave conical structure - detailed image. TESCAN $\operatorname{MIRA3}$ 


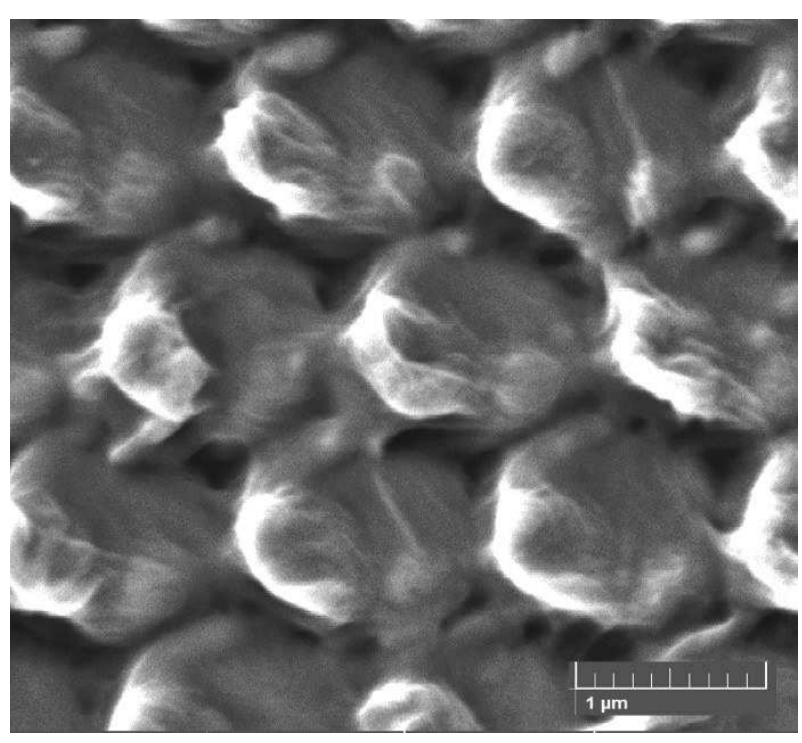

Fig. 15 The polycaprolactone (PCL) replica of pattern - convex conical structure - detailed image. TESCAN MIRA3

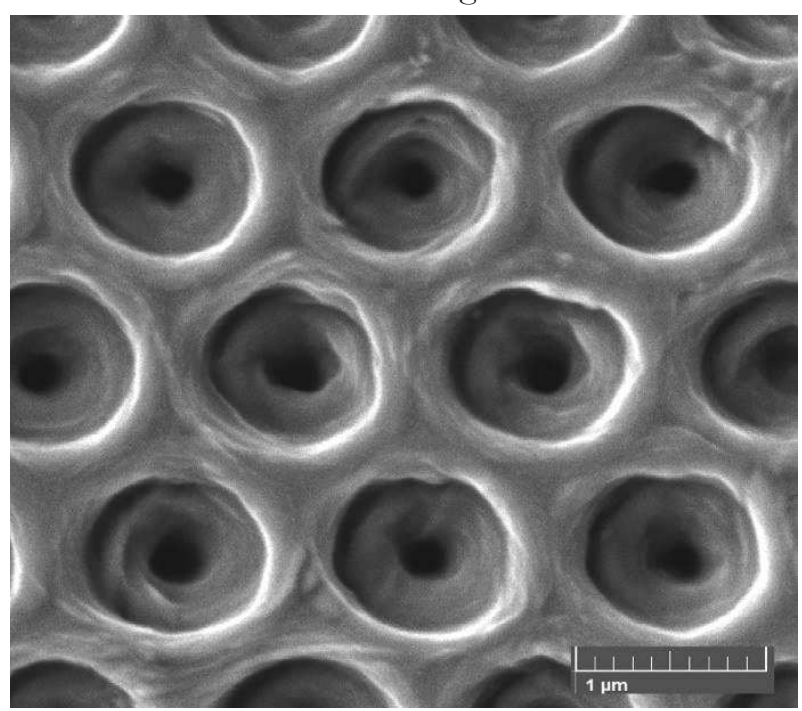

Fig. 16 The polycaprolactone (PCL) replica of pattern concave conical structure - detailed image. TESCAN $\operatorname{MIRA3}$

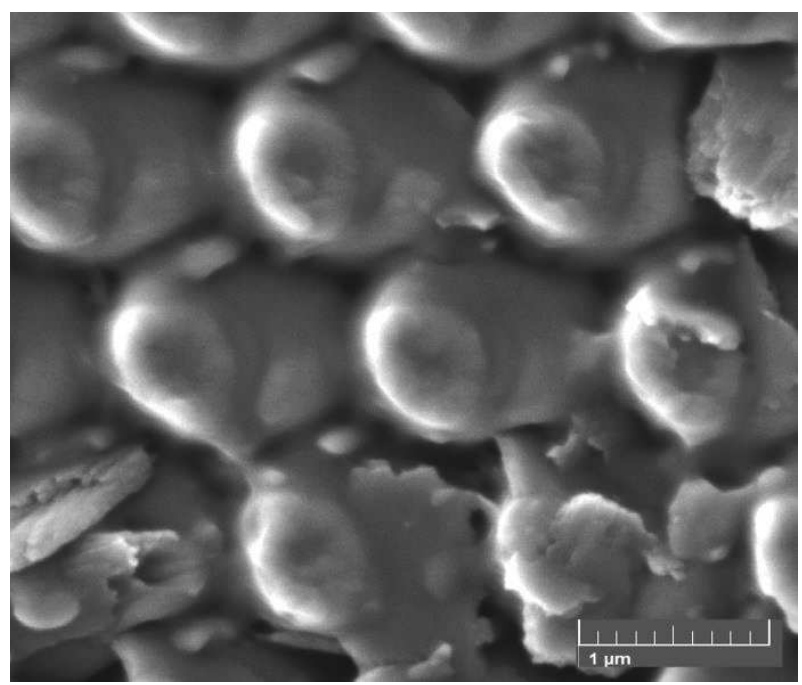

Fig. 17 The epoxy resin (EP) replica of pattern - convex conical structure - detailed image. TESCAN MIRA3

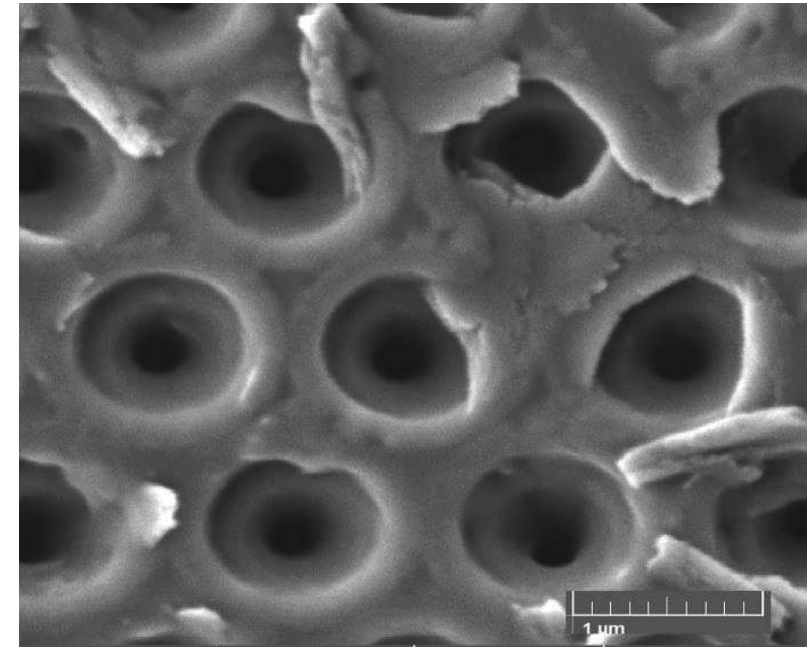

Fig. 18 The epoxy resin (EP) replica of pattern - concave conical structure - detailed image. TESCAN MIRA3

Polycaprolactone (PCL) is a crystallizing biodegradable polymer. It was applied to the silicone mold so that the melt formed by melting the PCL granule at a temperature of $80^{\circ} \pm 2^{\circ} \mathrm{C}$ was pressed into the silicone mold by a surface load of up to $5 \mathrm{~N}$. The PCL sample was cooled freely in a silicone mold for 20 minutes and then removed from the mold. The thickness of the PCL samples ranged from 1 to 2 millimeters. From the scanning electron microscope images - Figs. 15, Fig. 16 it is evident that this type of polymer is also able to form convex and concave replicas analogous to the original pattern. The fibrous structure on the surface of convex cones probably arises as a result of the process of crystallization of the polymer in the mold - Fig. 15. Concave cones copy the pattern on the silicon wafer well in shape, arrangement, and dimensions.

Epoxy resin (EP) belongs to the group of thermosets. This polymer was applied to the silicone mold after mixing the resin with the hardener in an adequate ratio according to the material sheet. The sample was left in the mold for 24 hours at a normal laboratory temperature of $22^{\circ} \pm 2{ }^{\circ} \mathrm{C}$ and normal atmospheric pressure. The sample was then removed from the mold. The thickness of the samples was around 2 to 3 millimeters. From the scanning electron microscope images - Figs. 17, Fig. 18 it is evident that this type of polymer is also able to create convex and concave replicas, which by the arrangement of cones and their sizes correspond to the original pattern on the silicon wafer. When crosslinking the epoxy resin in silicone form, substructures were formed, which are evident in the detailed images - Fig. 17 and Fig. 18. This phenomenon can be explained by the fact that crosslinking of epoxy resin runs on the structured microscopic surface. As can be seen from Fig. 17, the resulting substructures have a fibrous character and are formed by nanoscale-shaped formations. Concave cones do not reach the quality of the original pattern, there are vi- 
sible surface substructures, the explanation is analogous, as in the case of convex cones. The structure of the resulting nanoforms has not been studied in more detail in this work.

\section{Conclusion}

Based on the evaluation of the performed experiments, it was found that the milling process of focused ion beam can prepare a structured surface that is analogous in shape to the natural surface. The structure on the silicon wafer was repeatedly replicated into the silicone elastomer without degrading it. The use of a silicone elastomer possesses a good ability to copy the formed structure from a silicon wafer and at the same time, it is easily removable.

Replication of the created pattern by the method of two-step imprinting by selected types of polymers was actualized on convex and concave structures. Different types of polymers show a different ability to copy the structure in detail. Convex conical replicas show surface structuring at the nanometer level for PVA, CMC, and PCL polymers. This phenomenon of water-soluble polymers PVA and CMC can be explained by its evaporation during the replication process in elastomeric form. In the case of PCL as a crystallizing polymer, the surface structuring can be explained by the crystallization process in soft elastomeric form. From the results of this study, it is clear the replication process is useful for the preparation of surfacestructured polymer samples at the micrometer and submicrometer levels. Each type of polymer exhibits specific behaviors related to its chemical composition and method of application to the elastomeric form. Surface structuring at the nanometer level on convex conical replicas may be of potential significance in further research as well as the applicability of surface reflectivity. Due to the dimensions of the cones of the proposed model, or the dimensions of the cones of convex and concave structures, silicon wafer, silicone mold, and all prepared polymer replicas also produced color on structured surfaces. The above-described formation of structures at the nanometer level on the surface of convex cones was not apparent when replicated by the same polymers in the case of cone dimensions of $30-40 \mu \mathrm{m}$. [9]

\section{Acknowledgement}

This work was supported by the Ministry of Education, Youth and Sports of the Czech Republic and the European Union - European Structural and Investment Funds in the frames of Operational Programme Research, Development and Education - project Hybrid Materials for Hierarchical Structures (HyHi, Reg. No. CZ.02.1.01/0.0/0.0/16_019/0000843). My thanks belong to Martin $\check{S}_{\mathrm{vec}}$ for microscopic analysis and Hana Tesařová and Michal Klášterecký from Tescan Orsay Holding for their support with realizing of experiments.

\section{References}

[1] BHUSHAN, B., JUNG, Y.C., KOCH, K. (2009). Micro-, nano- and hierarchical structures for superhydrophobicity, self-cleaning and low adhesion. In: Philos Trans A Math Phys Eng Sci, Vol. 367, No. 1894, pp. 1631 - 1672.

[2] SCHULTE, A.J., KOCH, K., SPAETH, M., BARTHLOTT, W. (2009) Biomimetic replicas: Transfer of complex architectures with different optical properties from plant surfaces onto technical materials. In: Acta Biomaterialia, Vol. 5, No. 6, pp.1848 - 1854.

[3] HUANG, Z., YANG, S., ZHANG, H., CAO, W. (2015). Replication of Leaf Surface for Light Harvesting. Scientific Reports, Vol. 5, No. 14281.

[4] HUANG, Z., CAI, C., KUAI, L., LI, T. HUTTULA, M., CAO, W. (2018). Leafstructure patterning for antireflective and selfcleaning surfaces on Si-based solar cell. In: Solar Energy, Vol. 159, pp. 733-741.

[5] KUBIŠOVÁ, M., PATA, V., SÝKOROVÁ, L., MALACHOVÁ, M. (2017). Use of cluster analysis for assessment of surface replicas machined by a laser beam. In: Manufacturing Technology, Vol. 17, pp. 489 - 493, ISSN 1213-2489.

[6] PODANÝ, J., TOMÍČEK, J. (2020). Analysis of small holes manufacturing for optomechanical components. In: Manufacturing Technology, Vol. 20, No. 2, pp.229 - 236. DOI: 10. 21062/mft.2020.036

[7] KAŠPAR, V., ROZLIVKA, J. (2020). Chemical degradation of 3D printed products. In: $M a-$ nufacturing Technology, Vol. 20, No. 1, pp. $45-48$. DOI: $10.21062 / \mathrm{mft} .2020 .010$

[8] DOBROCKÝ, D., STUDENÝ, Z., POKORNÝ, Z., JOSKA, Z., FALTEJSEK, P. (2019). Assessment of Surface Structure of Machined Surface. In: Manufacturing Technology, Vol. 19, No. 4, pp. 563 - 572. DOI: 10.21062/ujep/335.2019/a/1213-

$2489 / \mathrm{MT} / 4 / 563$

[9] KROISOVA, D. (2018). Natural Hierarchical Structures and Their Replication. In: NANOCON 2018: 10th Anniversary International Conference on Nanomaterials - Research and Application, pp. 306-311. Ostrava. TANGER. ISBN 9788087294895) 